\title{
Éditorial
}

\section{Pour de nouvelles études sur les acteurs religieux africains à l'ère du numérique}

\author{
Pamela Millet-Mouity', Frédérick Madore ${ }^{2}$
}

\section{Introduction}

Depuis l'étude pionnière d'O'Leary (1996) et surtout à partir des années 2000, tout un champ de recherche s'est développé sur la façon dont la religion s'inscrit dans les médias numériques - sites web, forums, blogues, médias de diffusion en ligne, réseaux sociaux, etc. - et les façons dont ceux-ci influent sur la manière dont les individus pratiquent leur religion. Nombreux sont les auteur.e.s qui ont travaillé sur des concepts permettant de mieux appréhender les nouvelles formes de religiosités apparues par l'intermédiaire du Web. Certains d'entre eux ont proposé le terme "cyberreligion » pour identifier de nouveaux types de rituels et de communautés religieuses dans le cyberespace. Les premières études ont eu tendance à isoler les manifestations religieuses sur Internet de celles plus «traditionnelles » à l'église, la mosquée ou au temple par exemple. Si Dawson (2000) a utilisé ce concept spécifiquement pour distinguer les organisations ou les groupes religieux qui n'existent qu'en ligne, Brasher (2001) a employé cette notion dans une perspective plus large désignant à la fois la présence des organisations et activités religieuses dans le cyberespace ainsi que les nouvelles pratiques, idées et sociabilités religieuses inspirées du Web qui ont émergé progressivement. Dans une étude consacrée aux cultes afro-américains dans le Nouveau Monde, Capone (1999) s'est par exemple intéressée à l'éclosion sur Internet d'une multiplicité de pratiques religieuses et de discours à teneur politique portés par les "cybersanteros », qui sont les initiés de différentes maisons de cultes existantes horsligne. Elle a ainsi montré comment le Web devient alors tout à la fois un espace privilégié de luttes idéologiques et de fabrication d'identités nouvelles au-delà des frontières de l'État-nation et des identités culturelles nationales, entre le global et la «tradition».

\footnotetext{
${ }^{1}$ Centre d'études en sciences sociales du religieux (CéSor). École des hautes études en sciences sociales (France)

${ }^{2}$ Centre interdisciplinaire de recherche sur l'Afrique et le Moyen-Orient (CIRAM). Université Laval (Canada).
} 
Voulant discerner les différentes formes de religiosité qui sont apparues en ligne, Helland (2000) a pour sa part proposé une autre formulation conceptuelle faisant la distinction entre "religion online», le type le plus fréquent où les rituels sont largement basés sur des pratiques hors ligne, et " online religion », dans laquelle la flexibilité d'Internet a permis la formation de nouvelles formes de religiosité et de pratiques religieuses vécues que sur le Web. Helland (2007) a cependant nuancé ses propres catégories analytiques en reconnaissant que la séparation entre les deux types est devenue de plus en plus ténue rejoignant ainsi l'analyse de Dawson et Cowan (2004). Cette distinction est néanmoins demeurée un outil important pour différencier les multiples stratégies employées par les groupes religieux et les utilisateurs dans leurs activités en ligne.

À partir de la seconde moitié des années 2000 et de la décennie suivante, la séparation entre ces deux catégories étant devenue de plus en plus ténue, un nouveau terme est apparu pour décrire la pratique religieuse en ligne dans le Web $2.0^{3}$, celle de « digital religion ${ }^{4} »$ ou « religion numérique» (Cheong et al., 2012). Pour Campbell (2012), ce terme ne réfère pas uniquement à la religion telle qu'elle est pratiquée et articulée en ligne, mais aussi à la façon dont les espaces numériques façonnent la pratique religieuse hors ligne et vice versa. Dans une recherche subséquente, Campbell (2017) a affirmé que les Digital Religion Studies sont devenues un champ d'étude à part entière, à l'intersection des Internet Studies and Media et des Religion, and Culture Studies, qui offre aux chercheurs des cadres utiles pour réfléchir aux façons dont les individus et les institutions religieuses négocient leurs vies religieuses " en ligne » et " hors-ligne » et leurs pratiques médiatiques. Si ce courant s'est particulièrement développé dans le monde anglo-saxon, les chercheurs francophones ont tardé à porter attention aux manifestations numériques du religieux (Douyère, 2015; Duteil-Ogata et al., 2015). Penser les différentes formes de religiosité des acteurs africains tant sur le continent que dans les diasporas à l'ère d'Internet implique de s'intéresser aussi bien à ces interconnexions qu'à la complexité des changements sociaux et culturels qui en découlent au sein de ce que Capone (1999) voyait déjà comme une « communauté en réseau » ou que Campbell (2015) nommera "religion en réseaux ».

Nombre de recherches empiriques portant sur les religions et les médias numériques ont émané de spécialistes des mondes musulmans. Pour Roy (2000), l'espace singulier qu'est Internet constitue un support qui facilite à la fois les interactions personnelles et l'atteinte de nouveaux auditoires. Dès lors, les messages islamiques se dé-

${ }^{3}$ Le Web 2.0 facilite notamment l'interaction entre utilisateurs. L'internaute devient acteur en alimentant les sites en contenu, comme les blogues et les réseaux sociaux, ou de manière collaborative.

${ }^{4}$ Ce terme a été utilisé comme titre de plusieurs colloques tels que l'International Conference on Digital Religion at the University of Colorado Boulder en janvier 2012 et le Digital Religion Symposium at the Donner Institute in Turku en Finlande en juin 2012. 
veloppent tout en sapant les modes traditionnels de l'autorité islamique et de l'interprétation religieuse (Anderson, 2003). Pour Bunt (2000, 2003, 2009), un des spécialistes les plus prolifiques sur le « cyber-islam », l'utilisation des médias numériques pour diffuser l'islam a engendré la prolifération de nouveaux réseaux de partage de connaissances collaboratifs où les utilisateurs - les «iMuslims $s^{5}$ »- sont invités à fournir des contributions défiant ainsi les modèles verticaux et traditionnels de l'autorité religieuse. C'est ce qu'il a appelé le "wiki-oriented Islam ».

L'entrée des acteurs religieux sur le Web a également été analysée sous la plume de spécialistes des christianismes. Tout en revenant sur les apports du modèle télévangéliste ${ }^{6}$, Mottier (2015) a étudié, à partir du cas d'une église de la région parisienne, la dialectique des rapports entre les Églises - et leurs leaders par extension -, les fidèles et les nouveaux moyens de communication afin de voir dans quelles mesures l'expérience (néo)pentecôtiste, et particulièrement l'action cérémonielle, se trouve modifiée, transformée, voire réinventée par la médiation des écrans. Par sa perspective anthropologique, l'auteur donne également à voir la complexité du processus de globalisation de cette expérimentation divine et de mise en scène des performances scéniques des leaders ainsi que les recompositions des modalités du croire qui se donnent à voir avec la figure du «télé-fidèle ${ }^{7}$ ». Dans le contexte d'une économie de la médiatisation numérique omniprésente, le Net est devenu un élément central du processus de socialisation religieuse et d'incorporation du divin chez les (néo)pentecôtistes.

Si Internet offre à l'acteur religieux « moderne » un vivier de possibilités, l'une des spécificités de son utilisation est fondée sur sa capacité à faciliter la prolifération d'une pluralité de pratiques religieuses désengagées et/ou anonymes sous couvert de pseudonymes voire d'avatars (Jonveaux 2007 ; 2013). Ceci interroge par extension les formes de ce que Hervieu-Léger $(1999$; 2003) nomme le «bricolage religieux », et plus particulièrement le processus de construction du sujet croyant et les nouvelles manières de croire développées par ces «croyants-connectés»(Millet-Mouity, 2018). L'univers catholique analysé par Jonveau $(2007$; 2013) est d'ailleurs tout à fait exemplaire des tensions persistantes autour de l'impersonnalité et du désengagement, et

\footnotetext{
${ }^{5}$ En référence aux produits de la compagnie Apple.

${ }^{6}$ Sur le modèle télévangéliste qui a émergé aux États-Unis dès le début des années 1920, voir notamment les travaux pionniers de Hadden et Swann (1981) et de Gutwirth (1987, 1998).

${ }^{7}$ Pour Mottier (2015), la figure du télé-fidèle renvoie à celui qui suit le culte et participe à l'expérience cultuelle qu'il engendre par écran interposé, dont il serait certainement intéressant d'analyser les postures corporelles significatives de sens - mains posées par exemple sur l'écran à la demande du pasteur -, qui occupent une position centrale dans les recompositions des modalités du croire.
} 
plus largement de l'efficacité symbolique des activités religieuses en ligne (réalisation des rituels par exemple).

Un autre aspect à considérer concerne les pratiques dites de papillonnage d'une église virtuelle, d'un site, forum ou réseau social à l'autre, en fonction des affinités électives et des attentes subjectives de chaque croyant-connecté, en dehors ou au sein même des communautés ou congrégations religieuses d'origine (Millet-Mouity, 2018). Mayer (2010) a ainsi souligné les apports de la Toile sur les logiques d'évangélisation et la mise en relation d'acteurs sociaux aux idées religieuses à la fois semblables et divergentes. Les recherches sur le lien entre Internet et l'univers religieux ont donc mis en évidence de quelles manières et selon quelles modalités les acteurs religieux, dans un double mouvement (en ligne et hors-ligne), se sont de fait adaptés à ce qu'on pourrait qualifier de " contrainte contemporaine ", dans une ère marquée, constate MilletMouity (2018), par les e-novations d'une économie très concurrentielle du «bien-êtresalut ».

D’autres études se sont concentrées plus spécifiquement sur le lien entre les religions et les réseaux sociaux tels que Facebook, Twitter et YouTube (Cheong et al., 2012; Gillespie et al., 2013). Il en est notamment ressorti l'idée que ces outils numériques ont ouvert la voie à de nouveaux acteurs, qui n'ont pas nécessairement le profil classique des autorités spirituelles traditionnelles - souvent des hommes d'âge mûr -, pour acquérir une légitimité voire une autorité religieuse. Wheeler (2014) a par exemple montré comment des femmes musulmanes vloggers ${ }^{8}$ sur YouTube redéfinissent et renégocient les discours et représentations dominantes sur les femmes qui pratiquent l'islam. D'autres auteur.e's ont aussi avancé que la consommation de contenus religieux sur Facebook par des croyants répond non seulement au besoin de développer leurs connaissances religieuses, mais est aussi associée à la détente, la distraction et au divertissement (Brubaker et Haigh, 2017). La question du rôle d'Internet comme outil de radicalisation, de propagande et de recrutement pour des groupes terroristes comme Al-Qaïda et Daesh a aussi fait l'objet de vifs débats parmi les chercheurs au cours des dernières années (Denning, 2011 ; Jarvis et al., 2015 ; Aly et al., 2017).

\section{Les études sur le religieux et le numérique en Afrique subsaharienne : un champ d'études à défricher}

La popularité du champ d'études qui s'est développé autour des médias et des groupes religieux au cours des vingt dernières années n'a pas épargné l'Afrique subsaharienne. La majorité des travaux publiés sur ce sujet ont mis l'accent sur les small media (cassettes audio, films vidéo, CD, DVD) et les médias de masse plus «tradition-

\footnotetext{
${ }^{8}$ Le vlogger est un blogueur vidéo. YouTube est la plateforme de prédilection des vloggers.
} 
nels » que sont la presse, la radio et la télévision - ce qui n’est pas surprenant compte tenu de leur dominance dans le paysage médiatique de cette région. Cependant, peu d'études ont jusqu'à présent traité de manière significative de l'usage des médias numériques par les groupes religieux subsahariens si bien que le continent africain est quelque peu resté en marge des Digital Religion Studies. Par exemple, seules deux des quinze contributions de l'ouvrage collectif New Media and Religious Transformations in Africa (Hackett et Soares, 2014) portaient spécifiquement sur ce type de médias (Asamoah-Gyadu, 2014; Bezabeh, 2014). Hackett avait d'ailleurs identifié les recherches sur les sites Internet et les réseaux sociaux religieux comme des vides dans la littérature scientifique sur l'étude des religions et des médias africains, qui devaient être comblés d'urgence (Hackett et al., 2014). Pourtant, depuis le milieu des années 2000, les médias numériques sont devenus des outils, voire des espaces majeurs où se donne à voir le « religieux africain » en train de se faire dans sa forme plurielle.

Certes, le degré de pénétration et l'accessibilité d'Internet en Afrique demeurent parmi les plus faibles dans le monde : $31,2 \%$ de la population y avait accès selon des chiffres de juin $2017^{\circ}$. Malgré tout, son usage est en forte augmentation : entre 2000 et 2017, le nombre d'utilisateurs sur le continent africain est passé de 4500000 à plus de 388000 000, soit une croissance de 8503,1\%! Il y aurait également aujourd'hui plus de 160000000 comptes africains sur Facebook. Derrière ces statistiques se cachent toutefois d'importantes disparités entre l'Afrique du Nord et l'Afrique subsaharienne, entre les différentes régions linguistiques - le taux de pénétration atteint $89,4 \%$ au Kenya et $47,7 \%$ au Nigéria alors qu'il se situe entre $12 \%$ et $26,5 \%$ pour les pays d'Afrique de l'Ouest francophone - ainsi qu'entre les zones urbaines et rurales.

Les premières études sont surtout venues de spécialistes des églises évangéliques et (néo)pentecôtistes en Afrique de l'Ouest anglophone, dont leurs groupes et figures ont été initialement les plus entreprenants parmi les différentes confessions. AsamoahGyadu (2007, 2014) a montré comment les églises charismatiques (néo)pentecôtistes du Ghana se sont rapidement appropriées Internet comme un outil majeur d'évangélisation tant à l'échelle nationale que transnationale ainsi que de promotion du charisme de leurs leaders. Ceci rejoint les conclusions de Mayer (2010), qui avait également décelé des formes de sacralisation du cyberespace dans les recours à Internet par certains catholiques contemporains. Cependant, Asamoah-Gyadu a observé que les dénominations chrétiennes «traditionnelles» du Ghana (presbytériens, méthodistes, catholiques et anglicans) se sont longtemps contentées d'une utilisation limitée d'Internet : simplement comme une source d'information sur leurs activités, ce qui s'apparente donc à la « religion online » pour reprendre la terminologie d'Helland (2000, 2007). Hackett (2009) allant dans le même sens qu'Asamoah-Gyadu, a aussi té-

\footnotetext{
${ }^{9}$ «Internet Usage Statistics for Africa », Internet World Stats. Consulté le 2 novembre 2017, URL : www.internetworldstats.com/stats1.htm.
} 
moigné de l'importance croissante de la Toile comme espace important pour la constitution du leadership pentecôtiste dans les contextes africains et diasporiques. Elle a montré comment l'aspect multimédia d'Internet peut permettre efficacement de promouvoir le charisme d'un leader autant par le biais du support visuel, textuel ou audio.

D’autres recherches ont aussi porté sur l'utilisation des médias numériques par les autres confessions religieuses. Étudiant les usages d'Internet par l'Église catholique au Cameroun et ses fidèles, Mouthé (2015) a affirmé que le Web a reconfiguré le rapport à la foi, à l'Église et à la communauté en favorisant notamment une forme d'individualisme religieux. Quant à Hassane (2009), il avance que l'emploi d'Internet par les musulmans nigériens contribue au développement d'un espace public islamique et à l'émergence d'une société civile islamique qui s'approprie les codes, les règles et les outils de la globalisation marchande pour les appliquer à la prédication islamique. Pour Hassane (2009), le Web rend possible une « forme de bricolage idéologique qui consiste à composer un discours islamique, une argumentation, par la juxtaposition de discours et d'informations politiques provenant des quatre coins de la planète » (p. 116). Madore (2016) a pour sa part montré comment le développement de la da'wa (prosélytisme) numérique au Burkina Faso et en Côte d'Ivoire, bien qu'encore embryonnaire, a permis à de jeunes musulmans, plus à l'aise avec ces technologies, d'acquérir une légitimité auprès de leurs aînés, tout en offrant à des autorités religieuses établies et plus âgées de nouvelles possibilités pour réaffirmer leur influence. L'auteur a également avancé que cette nouvelle donne a favorisé la transnationalisation de l'identité islamique locale et la plus grande diffusion d'une conception littérale, voire salafiste, de l'islam détachée des enjeux politiques. Ces usages du numérique par des musulmans ouest-africains, bien qu'ils restent façonnés par le contexte local et national, font ainsi écho aux grandes dynamiques observées ailleurs dans les mondes musulmans tel que soulignées par les recherches évoquées précédemment.

\section{Les contributions du numéro}

Bien qu'elles soient encore peu nombreuses dans le contexte de l'Afrique subsaharienne, les premières études ont souligné la nécessité d'entreprendre des recherches supplémentaires, tant empiriques que théoriques, compte tenu de l'imbrication des médias numériques et de la religion ainsi que la rapidité de leurs transformations macro et micro. Les auteur.e.s des six articles de ce numéro thématique ont cherché à y contribuer en réfléchissant à l'utilisation des médias numériques par divers groupes religieux et figures charismatiques dans cinq pays (Burkina Faso, Sénégal, Côte d'Ivoire, Cameroun, République démocratique du Congo).

Ce numéro spécial entend rendre compte de la manière dont la présence du religieux sur Internet permet d'interroger aussi bien les institutions, les communautés, 
les croyances, les pratiques que les subjectivités religieuses. Il s'agit de porter un regard pluridisciplinaire sur les nouvelles réalités induites par l'irruption des médias numériques dans la sphère religieuse des mondes africains. Ainsi, chaque contribution questionne, à sa façon, les multiples bricolages, ramifications, circulations et conflictualités qui travaillent le « religieux africain » à l'ère du numérique, tant au niveau des discours et des représentations que des pratiques. Les auteur.e.s s'attachent non seulement à interroger les effets de l'utilisation d'Internet par les acteurs religieux sur les manières de croire, de faire croire et les subjectivités qui en découlent, mais également à explorer les articulations fines entre ces deux sphères - « religieux africain » et médias numériques - à partir de terrains inédits. Qu'il s'agisse d'analyser le contenu des pages Facebook, comptes Twitter et forums de discussions de certaines associations ou figures religieuses parmi les plus actives et les plus populaires ou encore de réaliser des enquêtes auprès des responsables et des fondateurs de quelquesuns des principaux sites web religieux, ce numéro spécial se veut donc une contribution originale à une région qui reste sous-étudiée par rapport au reste du monde et notamment le monde occidental.

Le numéro s'ouvre sur un article de Koudbi Kaboré, qui a étudié les usages d'Internet au sein de l'Union fraternelle des croyants (UFC), un mouvement interreligieux implanté à Dori, une ville à majorité musulmane du nord du Burkina Faso. Dans un contexte régional et national marqué par un nombre croissant d'attaques terroristes, ce texte offre un regard intéressant qui fait le contrepoids aux analyses mettant l'accent sur la radicalisation, les conflits et les tensions entre différentes confessions religieuses. En effet, les médias numériques, tels qu'employés par l'UFC, sont un outil important au service du dialogue et de la paix entre les catholiques et les musulmans du pays.

La deuxième contribution, de Sergine Sylla, porte sur l'utilisation du Web par les Mourides. Cette confrérie sénégalaise est réputée pour avoir su articuler foi et plateformes numériques comme aucune autre du pays n’a pu le réaliser (Bèye, 2011 ; Bondaz, 2015). À travers le cas de Hizbut-Tarqiyyah, Sylla avance que les médias numériques confessionnels mis en place par ce groupe contribuent non seulement à la diffusion de la culture et de l'identité du mouvement religieux, mais peuvent aussi être considérés comme de nouveaux lieux de culte montrant ainsi l'imbrication croissante des sphères religieuses en ligne et hors-ligne.

S'appuyant sur ses recherches menées en Côte d'Ivoire, Issouf Binaté s'intéresse aux façons dont les musulman·e·s ivoiriens investissent Facebook, le réseau social le plus populaire du pays. À travers l'analyse de messages, photos et vidéos publiés sur différents groupes Facebook et pages de certaines des principales figures musulmanes, l'auteur étudie les stratégies prosélytes et mobilisatrices de ces musulman·e·s désireux de participer aux débats sociopolitiques de leur pays. Dans un contexte national marqué par des tensions électorales et de nombreuses fractures communautaires ces dernières années, Binaté montre que les activités en ligne de ces croyant·e·s se tradui- 
sent en un ingénieux mélange de genres ainsi qu'en un langage hybride, entre prosélytisme religieux, divertissement et action militante sur le terrain du maintien de la paix sociale.

Dans une tout autre perspective, Louis Audet Gosselin interroge pour sa part les activités des Églises chrétiennes de Ouagadougou, au Burkina Faso, sur les réseaux sociaux. L'auteur examine notamment le rôle joué par les gatekeepers chrétiens dans la régulation et la diffusion des messages catholiques et évangéliques qui circulent sur le Web. Bien que ces pratiques communicatives chrétiennes nourrissent des échanges entre adhérent-e.s burkinabés et croyants ailleurs dans le monde, elles tendent à favoriser l'existence d'un espace médiatique unifié selon Audet Gosselin. Malgré la pluralité des acteur.trice-s, les voix discordantes demeurent marginales sur Internet occultant ainsi tout un pan des tensions inhérentes à la sphère chrétienne dans la société burkinabè.

Dans le cadre d'enquêtes de terrain menées entre 2013 et 2017, Sariette et Abia Batibonak se penchent sur la "modernité néopentecôtiste » à travers l'utilisation des médias numériques par les (néo)pentecôtistes dans les villes de Douala et Yaoundé. Les auteur.e-s étudient des manières de croire et de faire croire peu investies par les spécialistes des pentecôtismes, en particulier par ceux qui s'intéressent aux formes que prend cette religiosité dans les sociétés d'Afrique subsaharienne. En mettant l'accent sur les subjectivités des «fidèles à distance » - ceux qui participent aux cultes de guérison-délivrance et qui suivent en direct ou en différé les shows de prédicateurs africains célèbres via YouTube et d'autres médias numériques -, Batibonak et Batibonak explorent les innovations et les e-novations des (néo)pentecôtistes. Ils montrent ainsi comment ces processus imaginatifs et créatifs s'inscrivent plus largement dans l'ambition de ces croyanteess en perpétuelle quête de renouveau à vouloir « changer le monde » à partir des églises.

Enfin, l'article de Didier Makal questionne l'impact des médias numériques et des acteurs religieux dans les affrontements politiques qu'a connus la République démocratique du Congo au cours des dernières années. Dans un contexte national encore marqué par les effets de crises successives, dont la guerre civile de 1998-2002, et l'expiration du dernier mandat constitutionnel du président Joseph Kabila en décembre 2016, Makal offre une grille de lecture intéressante des tensions politiques et religieuses qui se sont exprimées sur le Web. L'auteur montre à quel point sous l'apparence « anodine » d'opinions et d'analyses contradictoires, les discours religieux diffusés sur Internet participent à une régulation du politique par le religieux relevant tantôt de la contestation ou de la légitimation du pouvoir en place.

Bien que les contributions traitent de différentes confessions et soient marquées par une diversité de perspectives méthodologiques et analytiques, plusieurs thématiques transversales sont communes aux différentes études de cas tel que l'a relevé Katrien Pype dans les réflexions qu'elle propose en conclusion du numéro. Qu'il s'agisse de l'importance des «réseaux religieux » ou des «interactions entre le reli- 
gieux et la politique », ou bien encore des «pratiques de foi en ligne et leur dialectique avec la politique de la religion », Pype s'est inspirée de ses propres travaux empiriques menés dès 2003 dans le monde médiatique Kinois pour formuler ses réflexions conclusives. Les analyses réalisées par les auteurs concordent donc sur le constat fait par Pype d'un enchevêtrement des mondes religieux et médiatiques.

Le numéro propose également un entretien avec Rosalind I. J. Hackett, une des pionnières dans les recherches sur les religions et les médias en Afrique. Déjà en 2005 , elle avait suggéré un cadre théorique pour analyser la "multifonctionnalité de l'internet en matière de religion » (Hackett 2005 : 86). Depuis, elle s'est beaucoup intéressée sur le pluralisme religieux et les questions entourant les médias, le genre et les religions sur le continent africain et notamment en Afrique de l'Ouest anglophone. Se basant sur les mouvements charismatiques pentecôtistes et les religions traditionnelles africaines, elle identifie certaines tendances qui caractérisent l'utilisation des médias numériques par les groupes religieux d’Afrique subsaharienne. Elle propose également des pistes de réflexion ainsi que des conseils méthodologiques et théoriques à l'intention de chercheur·e·s s'intéressant à ces questions.

\section{Bibliographie}

ANDERSON J. W. (2003), « The Internet and Islam's New Interpreters », in D. F. EICKELMAN, J. W. ANDERSON (dir.), New Media in the Muslim World. The Emerging Public Sphere, Bloomington, Indiana University Press, p. 45-60.

Aly A., Macdonald S., Jarvis L., Chen T. M. (2017), « Introduction to the Special Issue: Terrorist Online Propaganda and Radicalization », Studies in Conflict e Terrorism, vol. 40, $\mathrm{n}^{\circ} 1$, p. $1-9$.

ASAMOAH-GYADU J. K. (2007), « “'Get on the Internet!” Says the LORD’: Religion, Cyberspace and Christianity in Contemporary Africa », Studies in World Christianity, vol. $13, n^{\circ} 3$, p. 225-242.

ASAMOAH-GYADU J. K. (2014), " "We Are on the Internet”: Contemporary Pentecostalism in Africa and the New Culture of Online Religion », in R. I. J. HACKETT, B. F. SOARES (dir.), New Media and Religious Transformations in Africa, Bloomington, Indiana University Press, p. 157-170.

BÈYE C. (2011), « Les mourides du Sénégal face aux défis des nouvelles technologies, le cas d'Internet », Le Temps des médias, $\mathrm{n}^{\circ}$ 17, p. 111-117.

BezABEH S. A. (2014), « Living across Digital Landscapes: Muslims, Orthodox Christians, and an Indian Guru in Ethiopia », in R. I. J. HACKETT, B. F. SOARES (dir.), New Media and Religious Transformations in Africa, Bloomington, Indiana University Press, p. 266-283. 
BONDAZ J. (2015), « Images cultuelles et écrans mourides : deux cas de controverse iconographique et religieuse sur Internet », in F. DutEIL-Ogata, I. JONVEAUX,

L. KUCZYNSKI, S. NIZARD (dir.), Le religieux sur Internet, Paris, L'Harmattan, p. 261274.

BraSher B. E. (2001), Give Me that Online Religion, New York, John Wiley \& Sons.

BRUbaker P. J., HAigh M. M. (2017), « The Religious Facebook Experience: Uses and Gratifications of Faith-Based Content », Social Media + Society, vol. 3, n² 2,11 p.

BunT G. R. (2000), Virtually Islamic: Computer-Mediated Communication and Cyber Islamic Environments, Cardiff, University of Wales Press.

BunT G. R. (2003), Islam in the Digital Age: E-Jihad, Online Fatwas and Cyber Islamic Environments, Londres, Pluto Press.

BuNT G. R. (2009), iMuslims: Rewiring the House of Islam, Chapel Hill, University of North Carolina Press.

CAMPBell H. A. (dir.) (2012), Digital Religion: Understanding Religious Practice in New Media Worlds, New York, Taylor \& Francis.

CAMPBELl H. A. (2015), « Les relations entre religion en ligne et hors ligne dans une société en réseaux », in F. DuteIL-Ogata, I. JonveAux, L. KuczYnSKi, S. NizARD (dir.), Le religieux sur Internet, Paris, L'Harmattan, p. 95-124.

Campbell H. A. (2017), « Surveying Theoretical Approaches within Digital Religion Studies », New Media e Society, vol. 19, n 1, p. 15-24.

CAPONE S. (1999), « Les dieux sur le Net. L'essor des religions d'origine africaine aux États-Unis », L’Homme, nº 151, p. 47-74.

Cheong P. H., Nielsen P. F., Gelfgren S. (dir.) (2012), Digital Religion, Social Media and Culture: Perspectives, Practices and Futures, New York, Peter Lang Publishing.

DAWson L. L. (2000), « Researching Religion in Cyberspace: Issues and Strategies », in D. E. COWAN, J. K. HADDEN (dir.), Religion on the Internet: Research Prospects and Promises, New York, JAI Press, p. 25-54.

DAWSON L. L., CowAN D. E. (2004), «Introduction», in L. L. DAWSON, D. E. CowAN (dir.), Religion Online: Finding Faith on the Internet, New York, Routledge, p. 1-16.

DENNING D. (2011), « Terror's Web: How the Internet is Transforming Terrorism », in Y. JEWKes, M. YAR (dir.), Handbook of Internet Crime, Abingdon, Routledge, p. 194213.

DOUYÈRE D. (2015), « De la mobilisation de la communication numérique par les religions », ticessociété, vol. 9, nº 1-2. 
Duteil-Ogata F., Jonveaux I., Kuczynski L., Nizard S. (dir.) (2015), Le religieux sur Internet, Paris, L'Harmattan.

Gillespie M., Herbert D. E. J., GreEnhill A. (dir.) (2013), Social Media and Religious Change, Berlin, De Gruyter.

GutwiRTh J. (1987), « L'Église électronique américaine », Études, vol. 366, n 6, p. 823833.

GUTWIRTH J. (1998), L'Église électronique : la saga des télévangélistes, Paris, Bayard.

HACKetT R. I. J. (2005), « Religion et Internet », Diogène, vol. 211, nº 3, p. 86-99.

Hackett R. I. J. (2009), « The New Virtual (Inter)Face of African Pentecostalism », Society, vol. 46, nº, p. 496-503.

HACKeTt R. I. J., SOARES B. F. (dir.) (2014), New Media and Religious Transformations in Africa, Bloomington, Indiana University Press.

Hackett R., Mélice A., VAN Wolputte S., Pype K. (2014), « Interview: Rosalind Hackett Reflects on Religious Media in Africa », Social Compass, vol. 61, n 1, p. 67-72.

HadDen J. K., Swann C. E. (1981), Prime Time Preachers: The Rising Power of Televangelism, Reading, Addison-Wesley Publishing Company.

HASSANE S. (2009), « Société civile islamique : esquisse sur l'islam postmoderne et les pratiques religieuses "globales" en Afrique », in G. HOLDER (dir.), L'islam, nouvel espace public en Afrique, Paris, Karthala, p. 101-125.

HeLland C. (2000), « Online-Religion/Religion-Online and Virtual Communities », in D. E. COWAN, J. K. HADDEN (dir.), Religion on the Internet: Research Prospects and Promises, New York, JAI Press, p. 205-223.

Helland C. (2007), « Diaspora on the Electronic Frontier: Developing Virtual Connections within Sacred Homelands », Journal of Computer-Mediated Communication, vol. $12, n^{\circ}$ 3, p. 956-976.

HeRvieu-LÉGER D. (1999), Le Pèlerin et le converti. La religion en mouvement, Paris, Flammarion.

Hervieu-Léger D. (2003), Catholicisme, la fin d’un monde, Paris, Bayard.

Jarvis L., Macdonald S. K., Chen T. M. (dir.) (2015), Terrorism Online: Politics, Law and Technology, Londres, Routledge.

JONVEAUX I. (2007), « Une retraite de carême sur Internet », Archives de sciences sociales des religions, vol. 139, $\mathrm{n}^{\circ}$ 3, p. 157-176.

JonVeAux I. (2013), Dieu en ligne : expériences et pratiques religieuses sur Internet, Paris, Bayard. 
MADORE F. (2016), «L'islam ivoirien et burkinabé à l'ère du numérique 2.0 », Journal des anthropologues, $\mathrm{n}^{\circ}$ 146-147, p. 151-178.

MAYER J.-F. (2010), « Croire en ligne : usages religieux d'Internet et catholicisme contemporain », Transversalités, vol. 116, $\mathrm{n}^{\circ}$ 4, p. 45-62.

Millet-Mouity P. (2018), « D’une église à l'autre : phénoménologie du papillonnage religieux parmi les néo-pentecôtistes euro-africano-caribéens de la région parisienne », SociologieS, à paraître.

Mottier D. (2015), « Le télé-fidèle existe-t-il ? », in F. Duteil-Ogata, I. JonVEauX, L. KUCZYNSKI, S. NiZARD (dir.), Le religieux sur Internet, Paris, L'Harmattan, p. 173187.

MoutHÉ G. (2015), « Catholicisme et usages religieux de l'internet au Cameroun », ticessociété, vol. 9, nº 1-2.

O’LEARY S. D. (1996), « Cyberspace as Sacred Space: Communicating Religion on Computer Networks », Journal of the American Academy of Religion, vol. 64, $n^{\circ} 4$, p. 781808.

Roy O. (2000), « La communauté virtuelle. L’Internet et la déterritorialisation de l’islam », Réseaux, vol. 18, nº 99, p. 219-237.

WHEELER K. R. (2014), « Remixing Images of Islam. The Creation of New Muslim Women Subjectivities on YouTube », Online - Heidelberg Journal of Religions on the Internet, vol. 6, p. 144-163. 